\title{
Early onset life-threatening myelosuppression after low dose of intravesical thiotepa
}

\author{
Giancarlo Agnelli \\ M.D. \\ PaOlo Gresele \\ M.D.
}

Maria DE CunTo
M.D.

Albano del Favero

M.D.

\author{
Institute of Clinical Medicine, University of Perugia, 06100 Perugia, Italy
}

\begin{abstract}
Summary
Intravesical instillation of thiotepa is a popular treatment for localized bladder carcinoma. The drug can enter the systemic circulation, and such absorption may be enhanced by extensive tumour infiltration or by a recent tumour resection. A case is reported of early onset life-threatening pancytopenia following an unusually low dose of the drug in a patient who had undergone a recent tumour resection.
\end{abstract}

\section{Introduction}

The efficacy of intravesical instillation therapy with thiotepa $\left(\mathrm{N}-\mathrm{N}^{\prime}-\mathrm{N}^{\prime \prime}\right.$-thiethylene-thiophosphoramide), a poly-functioning alkylating agent, against bladder carcinoma was first reported by Jones and Swinney (1961). Subsequently, many clinical evaluations of this therapy have been performed regarding the direct destruction of and prophylaxis against bladder carcinoma (Abbassian and Wallace, 1966; Wescott, 1966; Drew and Marchall, 1968; Veenema, Dean and Uson, 1969; Pavone-Macaluso, Caramia and Rizzo, 1971; Tomiyama, 1972). At present, thiotepa remains a popular agent for the treatment of localized bladder carcinoma. Reduction in the extension of tumour or even complete eradication may be obtained in about one-third of the patients if they are treated with instillations of thiotepa (Prout, 1974).

The chief disadvantage of this therapy is the absorption of thiotepa into the circulating blood through the bladder wall. As with other alkylating agents, dose-related myelosuppression is a wellknown toxic effect, regardless of the route of administration. We report here a case of early onset lifethreatening pancytopenia following an unusually low dose of the drug.

Correspondence: Dr. Giancarlo Agnelli, Istituto di Clinica Medica, Policlinico Monteluce, 06100 Perugia, Italy.

\section{Case report}

A 68-year-old male was treated with 30-min intravesical instillations of thiotepa, $20 \mathrm{mg}$ weekly for $6{ }^{\circ}$ weeks, starting 20 days from resection of localized 을 malignant vesical papilloma. Before thiotepa treat- ment the patient had a normal blood cell count. $\subseteq$ White blood cell (WBC) and platelet counts performed before each weekly dose remained normal. Five days after the sixth instillation, the patient cong $\infty$ plained of fever, epistaxis, gingival bleeding, multiple

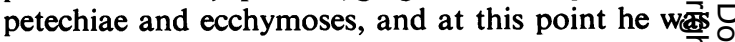
referred to our hospital.

Admission laboratory data showed a haematocrit $\frac{\bar{O}}{0}$ value of $17 \%$, a platelet count of $8 \times 10^{\circ} / 1$ and $\mathrm{a} \varrho$ WBC count of $0.7 \times 10^{9} / 1$, with a differential count $\stackrel{\Phi}{\varrho}$ of $2 \%$ neutrophils and $98 \%$ lymphocytes. Careful $\overrightarrow{\vec{P}}$ haemostatic examinations excluded disseminated $\frac{9}{3}$ intravascular coagulation. Bone marrow aspirate and $\bar{T}$ biopsy showed marrow aplasia. Chest X-ray showed bilateral pneumonia. Platelets and red cell concentrates were infused every day during the first week. Although antibiotic therapy was promptly started, $\dot{0}$ fever disappeared only after 8 days when the WBC 3 . count began to rise. Twenty days after admission, $\delta$ the WBC count was $2.7 \times 10^{\circ} / 1$ with $52 \%$ neutrophils. and the platelets $140 \times 10^{9} / 1$; the patient was dis-o charged.

\section{Discussion}

Myelosuppression has long been recognized as a consequence of intravesical instillation of thiotepan for bladder carcinoma. Nevertheless, this side effect N is often underestimated because of the erroneous $\sigma$ idea that intravesical thiotepa is a topical therapy rather than a systemic one.

Acute myelosuppression by intravesical thiotepa is dose related. The recommended dosage is usually $60 \mathrm{mg}$ thiotepa in $60 \mathrm{ml}$ distilled water instilled once $\bar{\circ}$ weekly. Two fatal cases of pancytopenia following $\overrightarrow{\mathbb{D}}$ 
such a treatment schedule have been reported by Abbassian and Wallace (1966) and an additional case has been reported by Bruce and Edgcomb (1967). Prout (1974) reduced both drug and diluent by $50 \%$ and observed salutary effects while decreasing the hazard of toxicity. Hollister and Coleman (1980), in a recent review of the haematological side effects of intravesical thiotepa therapy for bladder carcinoma, have suggested a guideline to minimize such toxicity. They recommended an initial treatment schedule averaging $90 \mathrm{mg} / \mathrm{month}$ and reported that only one of their 29 patients suffered pancytopenia with this dosage during the first three months. We have observed another case of early onset life-threatening pancytopenia following such a treatment schedule. In our patient, thiotepa therapy was started 20 days after resection of a localized malignant vesical papilloma. Absorption of thiotepa is greater in highly vascular tumours and through bladders in which there has been a recent resection (Koontz, 1979). The aforementioned data of Hollister and Coleman (1980) are incomplete regarding the extension of tumour and previous treatment.

In our opinion patients who have undergone a recent resection should be treated, at least for the first three months, with thiotepa doses lower than $90 \mathrm{mg} / \mathrm{month}$ and watched for signs of early toxicity. Frequent checks of blood cell count are mandatory, but they do not shelter the patient, as in the case we report, from early onset myelosuppression.

\section{References}

Abbassian, A. \& Wallace, D.M. (1966) Intracavitary chemotherapy of diffuse noninfiltrating papillary carcinoma of the bladder. Journal of Urology, 96, 461.

Bruce, E.W. \& Edgcomb, J.H. (1967) Pancytopenia and generalized sepsis following treatment of cancer of the bladder with instillations of triethylene thiophosphoramide. Journal of Urology, 97, 482.

Drew, J.E. \& Marchall, V.F. (1968) The effects of topical thiotepa on the recurrence rate of superficial bladder cancers. Journal of Urology, 99, 740.

Hollister Jr, D. \& Coleman, M. (1980) Hematologic effects of intravesicular thiotepa therapy for bladder carcinoma. Journal of the American Medical Association, 244, 2065.

JoNES, H.C. \& SwINNEY, J. (1961) Thio-TEPA in the treatment of tumours of the bladder. Lancet, ii, 615.

KooNTZ, Jr, W.W. (1979) Intravesical chemotherapy and chemoprevention of superficial, low grade, low stage bladder carcinoma. Seminars in Oncology, 6, 217.

Pavone-Macaluso, M., Caramia, G. \& Rizzo, F.P. (1971) The use of chemotherapeutic agents in the prophylaxis of papillary bladder tumours. Urology International, 26, 379.

Prout Jr, G.R. (1974) Genitourinary tract cancer: The bladder, In: Cancer Medicine (Ed by Holland, J.F. \& Frei, E.), p. 1670. Lee \& Febiger, Philadelphia.

TomiYama, T. (1972) Effects of intravesical instillation of antitumor agents on bladder cancer. Japanese Journal of Urology, 63, 497.

Veenema, R.J., Dean Jr, A.L. \& Uson, A.C. (1969) Thiotepa bladder instillations: therapy and prophylaxis for superficial bladder tumors. Journal of Urology, 101, 711.

WescotT, J.W. (1966) The prophylactic use of thio-tepa in transitional cell carcinoma of the bladder. Journal of Urology, 96, 913. 